\title{
Analyse du résonateur transverse électromagnétique en cage d'oiseau par la méthode des éléments finis
}

\section{NasrEddine BENAHMED*, Mohammed FEHAM et M'Hamed KHELIF}

Département d'Electronique, Faculté des Sciences de l'Ingénieur, Université de Tlemcen, B. P. 119, 13000 Tlemcen, Algérie.

(Reçu le 30 Octobre 2005, accepté le 15 Janvier 2006)

*Correspondance, courriel : N_Benahmed@yahoo.fr

\section{Résumé}

Dans cet article nous avons réalisé un outil numérique pour analyser les grandeurs électromagnétiques du résonateur transverse électromagnétique (TEM) en cage d'oiseau non chargé formé par n-éléments de lignes microrubans.

Cet outil numérique permet la détermination des paramètres primaires $[L],[C]$ et $[R]$, nécessaires pour la conception et la simulation de la réponse fréquentielle à l'entrée radiofréquence (RF) de sonde RIM (Résonance par Imagerie Médicale) utilisant le résonateur TEM en cage d'oiseau.

Comme application, nous présentons les résultats d'analyse obtenus par la Méthode des Eléments Finis (MEF) du résonateur à 12 éléments.

Mots-clés : sonde RIM, résonateur TEM en cage d'oiseau, paramètres [L], [C] et [R], méthode des éléments finis (MEF).

\section{Abstract}

Finite Element Analysis of The Transverse Electromagnetic Birdcage Resonator

In this paper we have successfully realized a numerical tool to analyze an n-element unloaded microstrip line transverse electromagnetic (TEM) resonator for magnetic resonance imaging. This numerical tool allows the determination of the primary parameters: $[L],[C]$ and $[R]$ matrices witch permit the design and the simulation of the frequency response at the RF port of the IRM probe.

As an application, we present the results obtained by Finite Element Method (FEM) analysis of a 12 elements.

Keywords : IRM probe, shielded birdcage resonator, parameters [L], [C] and [R],

finite element method (FEM). 


\section{Introduction}

L'extension actuelle de la résonance magnétique nucléaire (RMN) à de nombreux domaines aussi variés que la chimie (spectroscopie) et médecine (imagerie : RIM) a un caractère extrêmement dynamique.

Le développement des techniques cryogéniques a permis de produire des champs magnétiques $\overrightarrow{\mathrm{H}}_{0}$ très forts, très homogènes spatialement ef très stables dans le temps. Ces champs $\overrightarrow{\mathrm{H}}_{0}$ permettent l'accessibilité à de nombreux noyaux. Dans ces conditions, de nombreuses expériences (micro-imagerie, ...) de très haute homogénéité de $\overrightarrow{\mathrm{H}}_{0}$ et haute puissance, nécessitent la redéfinition de la sonde. La sonde est un circuit résonnant constitué d'un élément selfique (solénoïde, ligne à bandes symétriques, ligne en anneau fendu, tore, cage d'oiseau, ...) et d'autre composants pour assurer l'accord et l'adaptation. L'élément selfique que nous appellerons résonateur joue un rôle capital au niveau du spectromètre. Ce résonateur doit entre autre avoir un coefficient de qualité et une homogénéité du champ $\overrightarrow{\mathrm{H}}_{1}$ ne devant pas perturber l'homogénéité de $\overrightarrow{\mathrm{H}}_{0}$ dont la valeur est aussi grande que possible. Pour répondre à cette exigence, une solution s'avérant efficace, consiste à utiliser des tronçons de lignes (éléments à constantes réparties) au lieu des composants ordinaires (éléments à constantes localisées) [1-3]. Le résonateur transverse électromagnétique (TEM) en cage d'oiseau est tout indiqué pour ce genre de conception [3].

Des travaux [4] ont montré qu'aux fréquences de fonctionnement de 200 et $400 \mathrm{MHz}$ correspondant respectivement à des champs de $4.7 \mathrm{~T}$ et $9.4 \mathrm{~T}$, le résonateur TEM en cage d'oiseau peut produire une meilleure homogénéité du champ et un meilleur coefficient de qualité que le résonateur en cage d'oiseau classique, ayant pour conséquence d'améliorer la qualité d'image.

La première différence entre le résonateur TEM en cage d'oiseau et celui classique est la présence du blindage cylindrique (Figure 1) qui fonctionne comme élément actif du système, fournissant un chemin de retour pour les courants dans les conducteurs intérieurs. Dans le résonateur en cage d'oiseau classique, le blindage est une entité séparée démontée des éléments intérieurs, seulement reflétant les champs à l'intérieur du résonateur pour empêcher les pertes de rayonnement excessives. En raison de la présence du blindage, le résonateur TEM en cage d'oiseau se comporte comme une ligne de transmission longitudinale à plusieurs conducteurs [3]. A la différence du résonateur en cage d'oiseau classique, les conducteurs intérieurs du résonateur de type TEM ne possèdent pas des raccordements, mais se relient directement au blindage par les éléments capacitifs [3] (Figure 2). 


\section{Méthodologie}

\section{2-1. Description du Résonateur TEM en cage d'oiseau}

La Figure 1 présente la structure du résonateur TEM en cage d'oiseau non chargé constitué par n-éléments de lignes microrubans formant un cylindre, connectés à leurs extrémités au blindage au moyens de capacités [3].

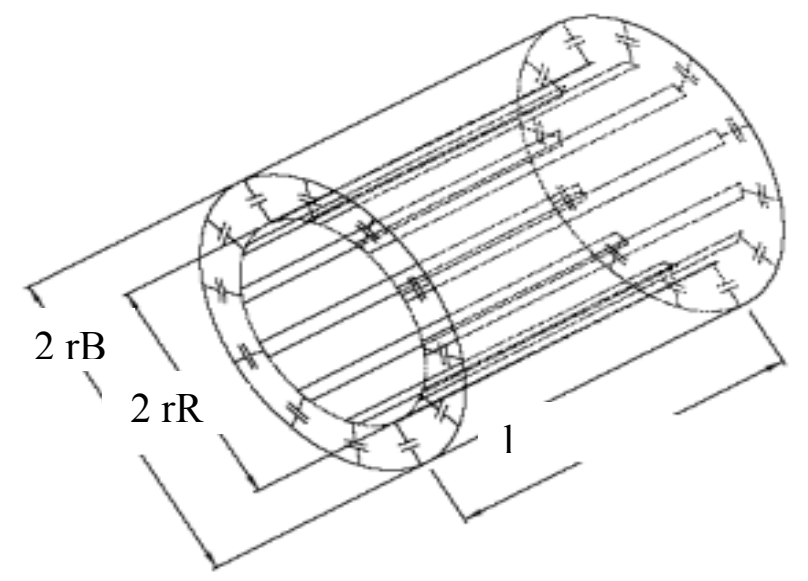

Figure 1 : Résonateur TEM en cage d'oiseau

La section transversale du résonateur TEM en cage d'oiseau est présentée sur la Figure 3. Elle est formée par un blindage extérieur de rayon $r B$ et $n$ éléments microrubans de largeur $w$ et d'épaisseur $t$ constituant un cylindre intérieur de rayon $r R$.

Le problème posé est un problème de Dirichlet avec une difficulté due aux contours des $n$ éléments ouverts et le domaine n'est pas simplement connexe. Pour l'analyse de ce type de géométrie, une solution analytique ef l'utilisation des transformations géométriques ne sont pas possibles.

Cependant les auteurs de la référence [3] ont utilisé la méthode BEM (Boundary Element Method). Dans ce papier, notre choix s'est porté sur la méthode des éléments finis qui est d'une utilisation plus souple [5]. 


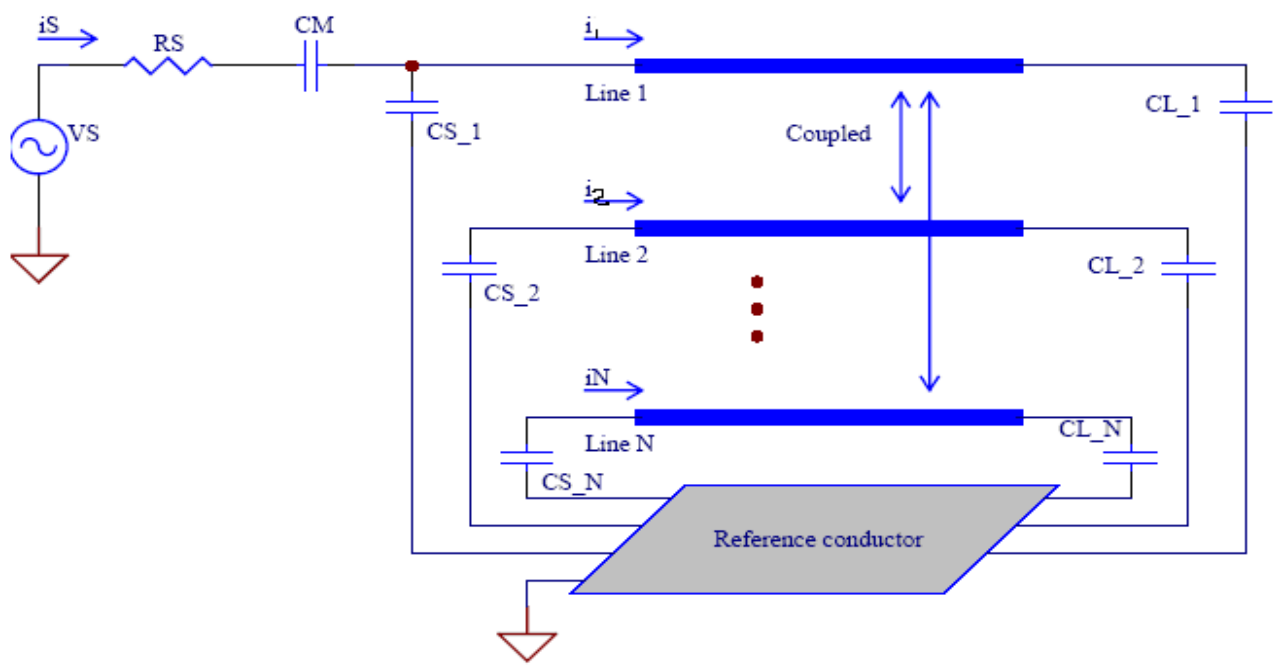

Figure 2: Schéma électrique du résonateur TEM en cage d'oiseau

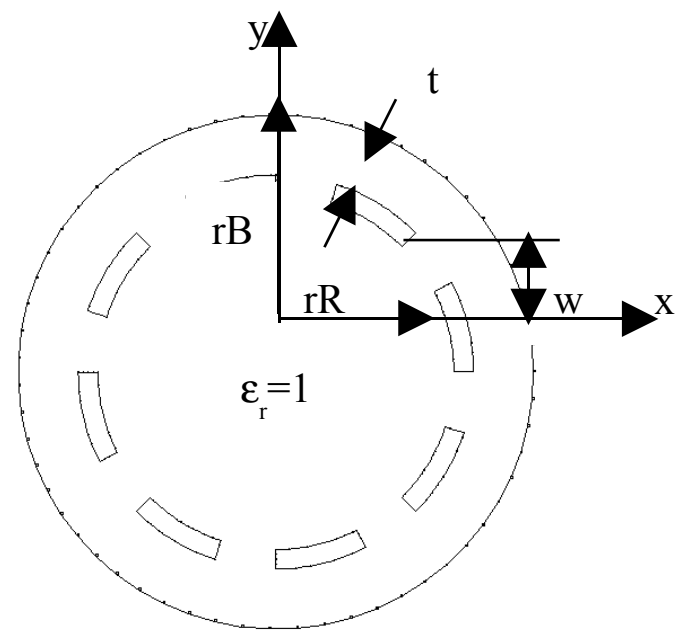

Figure 3: Section transversale du résonateur TEM en cage d'oiseau

\section{2-2. Détermination des paramètres électriques}

Les propriétés électromagnétiques du résonateur TEM en cage d'oiseau peuvent être décrits en termes de paramètres primaires $[\mathrm{L}],[\mathrm{C}]$ et $[R]$. 


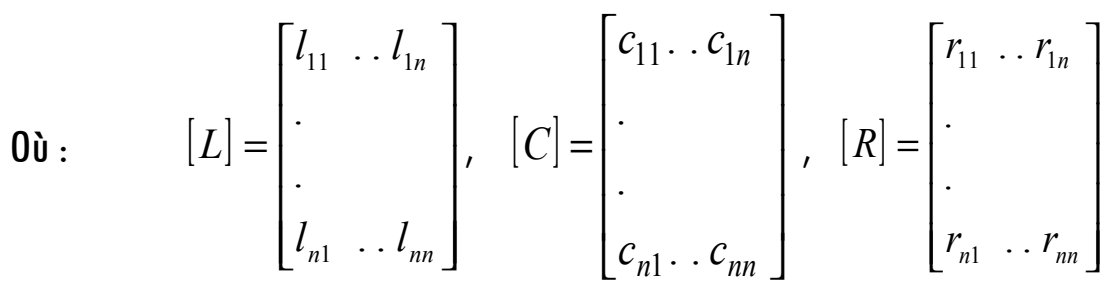

Les coefficients de ces matrices sont obtenus à partir de la résolution à deux dimensions de l'équation de Laplace $[3,6,7]$.

$$
\operatorname{div}\left[\nabla_{t} V(x, y)\right]=0
$$

Où :

$$
\begin{aligned}
& V=1 \text { V sur un conducteur } \\
& V=0 \text { V sur les autres conducteurs. }
\end{aligned}
$$

La solution de l'équation (1) représente la distribution du potentiel $V$ aux différents nœuds de maillage de la structure. Dans ce qui suit, nous présentons la méthode de calcul des coefficients des matrices $[\mathrm{L}],[\mathrm{C}]$ et [R].

\section{2-2-1. Calcul de la matrice capacité [8]}

Si un générateur est placé entre le résonateur et la masse, les courants et les charges sont excités dans la masse et dans le résonateur. La variation du courant induit une force électromotrice dans tous les conducteurs, et induit un couplage électromagnétique entre ces conducteurs.

Le couplage capacitif est décrit par l'équation électrostatique suivante :

$$
\begin{aligned}
& \mathrm{q}_{1}=\mathrm{C}_{11} \cdot \mathrm{V}_{1}+\mathrm{C}_{12} \cdot \mathrm{V}_{2}+\ldots+\mathrm{C}_{1 \mathrm{n}} \cdot \mathrm{V}_{\mathrm{n}} \\
& \mathrm{q}_{2}=\mathrm{C}_{21} \cdot \mathrm{V}_{1}+\mathrm{C}_{22} \cdot \mathrm{V}_{2}+\ldots+\mathrm{C}_{2 \mathrm{n}} \cdot \mathrm{V}_{\mathrm{n}} \\
& \cdot \\
& \cdot \\
& \mathrm{q}_{\mathrm{n}}=\mathrm{C}_{\mathrm{n} 1} \cdot \mathrm{V}_{1}+\mathrm{C}_{\mathrm{n} 2} \cdot \mathrm{V}_{2}+\ldots+\mathrm{C}_{\mathrm{nn}} \cdot \mathrm{V}_{\mathrm{n}}
\end{aligned}
$$

Où :

$q_{1}, q_{2}, \ldots, q_{n}$ sont les charges par unité de longueur (Coulomb/m) dans les conducteurs.

$V_{1}, V_{2} \ldots, V_{n}$ sont les potentiels (en Volt) entre les conducteurs et la référence.

$C_{1}, C_{2}, \ldots, C_{n}$ sont les capacités par unité de longueur $(F / m)$.

La charge par unité de longueur dans la masse est : $-\left(q_{1}+q_{2}+\ldots+q_{n}\right)$. Pour calculer les coefficients de capacité, on porte le conducteur 1 au potentiel $V_{1}$, et le reste des conducteurs au potentiel 0 ; il vient :

$$
c_{11}=q_{1} / V_{1}, \quad c_{21}=q_{2} / V_{1}, \ldots . ., \quad c_{n 1}=q_{n} / V_{1} .
$$


Si $V_{1}>0$ et $q_{1}>0$, alors $C_{11}>0$, d'où : $C_{m l}<0, m=2, . ., n$ (puisque ces conducteurs sont portés au potentiel nul).

De façon générale, on porte à chaque fois le conducteur $k$ ( $k$ variant de 1 à $n$ ) à un potentiel $V_{k}$ et le reste des conducteurs à un potentiel nul, puis on détermine les coefficients $C_{m k}$ et tous les coefficients $C_{k k}, m=1, . ., n$.

Le système d'équation (2) peut être écrit alors sous la forme matricielle suivante :

$$
[\mathrm{Q}]=[\mathrm{C}] \cdot[\mathrm{V}]
$$

avec:

$$
\begin{aligned}
& {[Q]=\left[\begin{array}{llll}
q_{1} & q_{2} & \ldots & q_{n}
\end{array}\right]^{\top}} \\
& {[\mathrm{V}]=\left[\begin{array}{llll}
\mathrm{V}_{1} & \mathrm{~V}_{2} & \ldots & \mathrm{V}_{\mathrm{n}}
\end{array}\right]^{\top}} \\
& \text { et } \quad[C]=\left[\begin{array}{l}
c_{11} \ldots c_{1 n} \\
. \\
c_{n 1} \ldots c_{n n}
\end{array}\right]
\end{aligned}
$$

[Q] est le vecteur de charge par unité de longueur,

[V] est le vecteur potentiel,

[C] est la matrice des coefficients de capacité par unité de longueur.

Les termes diagonaux représentent les capacités des lignes et les autres termes représentent les capacités de couplage entre deux conducteurs $(m, n)$ de valeur $-C_{m n} \Delta x(\Delta$ $x$ est la longueur du résonateur). (es capacités provoquent une chute de tension le long du résonateur.

\section{2-2-2. Calcul de la matrice inductance [8]}

On considère dans ce cas le couplage inductif. La variation du courant $\left(i_{1}, i_{2}, . ., i_{n}\right)$ dans les conducteurs et dans la masse $\left(i_{1}+i_{2}+. .+i_{n}\right)$ induit un champ électrique (force électromotrice), suivant la loi de Faraday qu'on peut écrire :

$$
\begin{array}{r}
e_{1}=-l_{11} \frac{\partial i_{1}}{\partial t}-\ldots-l_{1 n} \frac{\partial i_{n}}{\partial t} \\
e_{2}=-l_{21} \frac{\partial i_{1}}{\partial t}-\ldots-l_{2 n} \frac{\partial i_{n}}{\partial t} \\
\ldots \ldots \ldots \ldots \ldots . . \\
e_{n}=-l_{n 1} \frac{\partial i_{1}}{\partial t}-\ldots-l_{n n} \frac{\partial i_{n}}{\partial t}
\end{array}
$$


Où :

$I_{11}, I_{21}, . ., I_{n n}$ sont les inductances par unité de longueur $(\mathrm{H} / \mathrm{m})$ des éléments du résonateur. Par conséquent, l'équation (4) peut être écrite sous la forme matricielle suivante :

$$
[E]=-[L] . \partial[l] / \partial t
$$

Où :

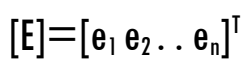

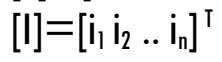

$$
\begin{aligned}
& {[L]=\left[\begin{array}{lll}
l_{11} & \ldots & l_{1 n} \\
\cdot & \\
\cdot & \\
l_{n 1} & \ldots & l_{n n}
\end{array}\right]}
\end{aligned}
$$

[E] est le vecteur de force électromotrice induite par unité de longueur (Volt/m). [I] est le vecteur courant (Ampère).

[L] est la matrice d'inductance par unité de longueur $(H / m)$. Ses termes diagonaux représentent les inductances propres des éléments du résonateur et les autres termes correspondent aux inductances mutuelles entre ces éléments. Dans le cas où les fréquences sont très élevées et les pertes sont négligeables, tous ces termes sont positifs.

Dans le circuit électrique équivalent, l'effet inductif dans le résonateur de longueur $\Delta$ $x$ est modélisé par l'introduction d'une inductance dans chaque élément (d'induction : $I_{\mathrm{mm}}$. $\Delta \mathrm{x}$ ) et toutes ces inductances sont mutuellement couplées.

Comme le résonateur est vide, alors :

$$
[L] .[C]=\varepsilon_{0} \mu_{0}
$$

Ainsi on détermine la matrice $[C]$ par l'analyse du problème électrostatique du résonateur TEM vide et on calcule ensuite la matrice $[L]$ par:

$$
[\mathrm{L}]=\left(\mathrm{l} / \mathrm{v}_{0}\right)^{2} \cdot[\mathrm{C}]^{-1}
$$

où :

$$
v_{0}=\frac{1}{\sqrt{\varepsilon_{0} \mu_{0}}} \text { est la vitesse de l'onde dans le vide. }
$$

L'analyse électrostatique permet aussi de déterminer la densité de courant de surface ( $\vec{J}_{S}$ ) par la relation : 


$$
\vec{J}_{s}=v_{0} \cdot \rho_{s 0} \cdot \vec{u}_{x}
$$

Où :

$\rho_{s 0}$ est la densité de charge de surface,

$\vec{u}_{x}$ est le vecteur unitaire de la direction longitudinale des éléments du résonateur.

\section{2-2-3. Calcul de la matrice résistance [8]}

Dans le cas des résonateurs avec pertes, les pertes de conduction dépendent du courant de conduction et par conséquent de la profondeur de pénétration $\delta$ (effet de peau) exprimée par la relation suivante :

$$
\delta=\frac{1}{\sqrt{\pi \mu_{0} f \sigma}}
$$

Où $\sigma$ est la conductivité du conducteur et $f$ la fréquence de travail.

Dans ce cas, les pertes sur le conducteur sont évaluées par la méthode des perturbations [8]. Cette méthode consiste en premier lieu, à supposer que le résonateur est sans pertes, pour déterminer la distribution du courant de surface $J_{s}$ à partir des solutions électrostatiques de la ligne vide suivant la relation (8). Puis en introduisant les pertes, la puissance $P_{c}$ dissipée par unité de longueur par le conducteur est calculée par la relation (10):

$$
P_{c}=\oint_{\Gamma} R_{s}\left|J_{s}\right|^{2} d l
$$

$R_{s}$ est la résistance de surface du conducteur, donnée par :

$$
R_{s}=\sqrt{\frac{\pi \mu f}{\sigma}}
$$

$\Gamma$ indique le contour total de tous les conducteurs.

Dans le circuit électrique équivalent, les pertes de conduction dans le résonateur de longueur $\Delta \mathrm{x}$ sont modélisées par des résistances en série dans chaque élément du résonateur et des résistances traduisant le couplage mutuel. Ces résistances contribuent à la chute de tension le long du résonateur.

La matrice $[\mathrm{R}]$ décrivant les pertes de conduction s'écrit : $[R]=\left[\begin{array}{lll}r_{11} & \ldots & r_{1 n} \\ \cdot & & \\ \cdot & & \\ r_{n 1} & \ldots & r_{n n}\end{array}\right]$ 
L'évaluation de la matrice [R] est basée sur l'équation (10) et sur l'expression :

$$
P_{c}=\sum_{i=1}^{n} \sum_{j=1}^{n} r_{i j}\left|I_{i}\right|\left|I_{j}\right|
$$

où : $\quad I_{i}$ est le courant du $i^{\text {ème }}$ conducteur.

En supposant $\mathrm{I}_{1}=1 \mathrm{~A}$ et tous les autres courants nuls, sachant que $\mathrm{P}_{\mathrm{c}}=\mathrm{r}_{11}\left|I_{1}\right|^{2}$, on détermine $r_{11}$. On répète la même procédure pour évaluer $r_{i i}$ pour $i=2$. ... Puis on prend $\mathrm{I}_{1}=\mathrm{I}_{2}=1 \mathrm{~A}$, et tous les autres courants nuls. Sachant que $\mathrm{r}_{12}=r_{21}$, on a dans ce cas $\mathrm{P}_{\mathrm{c}}=\mathrm{r}_{11}\left|I_{1}\right|^{2}+2 \mathrm{r}_{12}\left|I_{1}\right|\left|I_{2}\right|+\mathrm{r}_{22}\left|I_{2}\right|^{2}$. Connaissant tous les paramètres de cette équation, on détermine alors $\mathrm{r}_{12}$. On répète la même procédure pour évaluer tous les autres paires de courant $I_{i}$ et $I_{i}(i \neq i)$ pour obtenir le reste des éléments de la matrice $[R]$ qui est proportionnelle à $\sqrt{f}$ à cause de la relation (II).

La matrice $[R]$ est assimilée à une matrice inductance interne $\left[L_{i}\right]$ telle que :

$$
\omega\left[\mathrm{L}_{\mathrm{i}}\right]=[\mathrm{R}]
$$

où $\omega=2 \pi f$.

La matrice $\left[L_{i}\right]$ est additionnée à la matrice [L] par unité de longueur [8].

\section{Résultats et discussion}

Sur la base de cette théorie, un outil numérique utilisant la méthode des éléments finis a été réalisé pour déterminer les paramètres électromagnétiques du résonateur TEM en cage d'oiseau à 12 éléments.

Grâce à sa souplesse et la génération automatique du maillage (Figure 4), nous nous sommes intéressés au comportement du potentiel dans le résonateur ayant les caractéristiques suivantes [3], présentées dans le Tableav 1.

Tableau 1 : Caractéristiques géométriques et physiques du résonateur

\begin{tabular}{|c|c|}
\hline Paramètres & Valeurs \\
\hline Rayon du blindage $: \mathrm{rB}(\mathrm{mm})$ & 52.500 \\
\hline Rayon du cylindre intérieur $: \mathrm{rR}(\mathrm{mm})$ & 36.250 \\
\hline Largeurs des rubans $: \mathrm{w}(\mathrm{mm})$ & 6.400 \\
\hline Epaisseur des rubans $: \mathrm{t}(\mathrm{mm})$ & 0.038 \\
\hline Fréquence de travail $: \mathrm{f}(\mathrm{MHz})$ & 200 \\
\hline Conductivité $: \sigma(\Omega \mathrm{m})^{-1}$ & $5.8 \times 10^{7}$ \\
\hline Constante diélectrique $: \varepsilon_{\mathrm{r}}$ & 1 \\
\hline
\end{tabular}


La distribution du potentiel obtenue après résolution du problème est montrée sur la Figure 5. Ensuite, nous nous sommes intéressés à la distribution du potentiel pour les conditions d'excitation suivantes:

$$
\begin{aligned}
& V=1 V \text { sur les conducteurs ( } i=1 \text { à } 5) . \\
& V=-1 V \text { sur les conducteurs ( } i=7 \text { à } 11) . \\
& V=0 \text { sur les autres conducteurs. }
\end{aligned}
$$

Nous obtenons alors les résultats des Figure 5 et 6.

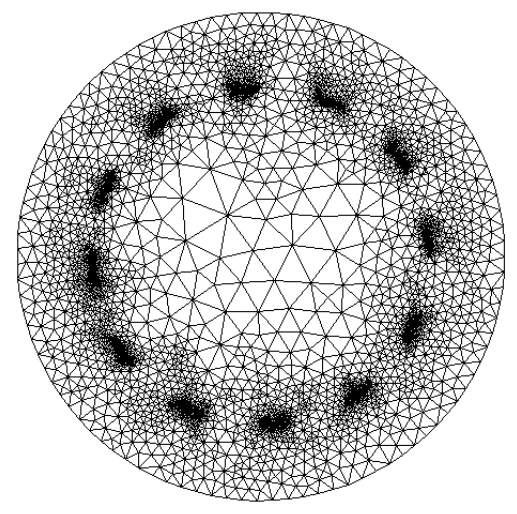

Figure 4 : Maillage de la structure

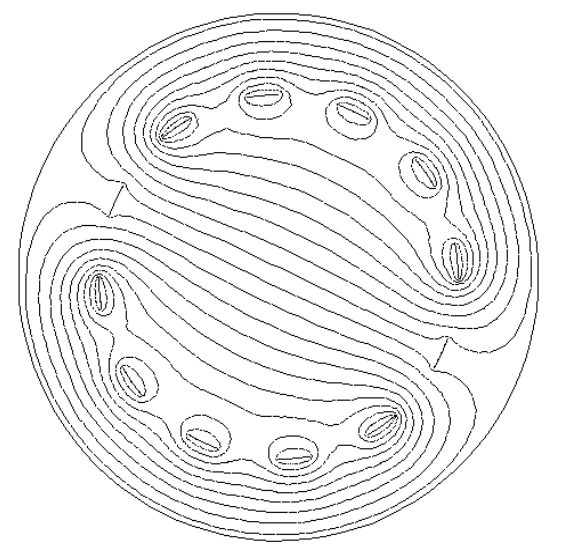

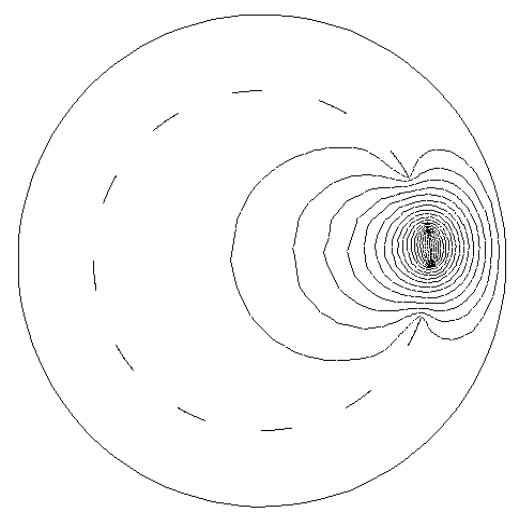

Figure 5 : Distribution du potentiel pour :

$V=1 V$ sur le conducteurs $(i=1)$ et $V=0$ sur les autres conducteurs

Figure 6 : Distribution du potentiel pour :

$V=1 V$ sur les conducteurs $(i=1 \grave{a} 5), V$ $=-1 V$ sur les conducteurs ( $i=7$ à 11$)$ et $V=0 V$ sur les autres conducteurs. 
Tableau 2 : Paramètres électriques du résonateur

\begin{tabular}{|c|c|c|c|c|c|c|}
\hline \multirow{2}{*}{ Colonne } & \multicolumn{2}{|c|}{$\mathrm{L}(\mathrm{nH} / \mathrm{m})$} & \multicolumn{2}{c|}{$\mathrm{C}(\mathrm{pF} / \mathrm{m})$} & \multicolumn{2}{c|}{$\mathrm{R}\left(10^{-3} \Omega / \mathrm{m}\right)$} \\
\cline { 2 - 7 } & MEF & $\begin{array}{c}\text { BEM } \\
{[3]}\end{array}$ & MEF & $\begin{array}{c}\text { BEM } \\
{[3]}\end{array}$ & $\mathrm{MEF}$ & $\begin{array}{c}\text { BEM } \\
{[3]}\end{array}$ \\
\hline 1 & 564.10 & 565.60 & 21.460 & 30.770 & 730.40 & 634.800 \\
& 116.70 & 116.70 & -3.896 & -6.551 & 14.790 & 17.900 \\
\hline 2 & 46.85 & 47.01 & -0.675 & -0.757 & 5.955 & 7.700 \\
\hline 1 & 25.86 & 25.96 & -0.306 & -0.330 & 5.766 & 6.400 \\
\hline 4 & 17.78 & 17.86 & -0.199 & -0.213 & 5.503 & 5.600 \\
\hline 5 & 14.45 & 14.51 & -0.158 & -0.169 & 5.233 & 5.100 \\
\hline 6 & 13.52 & 13.58 & -0.147 & -0.157 & 5.131 & 5.000 \\
\hline 7 & 14.45 & 14.51 & -0.158 & -0.169 & 5.233 & 5.100 \\
\hline 8 & 17.78 & 17.86 & -0.199 & -0.213 & 5.503 & 5.600 \\
\hline 9 & 25.86 & 25.96 & -0.306 & -0.330 & 5.766 & 6.400 \\
\hline 10 & 46.85 & 47.01 & -0.675 & -0.757 & 5.955 & 7.700 \\
\hline 11 & 116.70 & 116.70 & -3.896 & -6.551 & 14.790 & 17.900 \\
\hline 12 & & & & & & \\
\hline
\end{tabular}

Enfin, dans le Tableau 2 , nous avons confronté nos résultats numériques obtenus par la MEF, pour les caractéristiques géométriques et physiques indiquées précédemment, aux résultats numériques obtenus par la BEM.

A partir de ce tableau nous constatons clairement que l'écart est très faible entre les résultats obtenus par la MEF et ceux de la BEM.

\section{Conclusion}

Il s'agissait dans ce travail de caractériser numériquement le résonateur TEM en cage d'oiseau pour des applications IRM (conception et réalisation de sonde). Pour atteindre cet objectif, nous avons déterminé les paramètres électromagnétiques primaires du résonateur TEM. Or aux fréquences du domaine qui nous intéresse le problème s'approxime par la résolution de l'équation de Laplace. A cause de sa complexité due au type de géométrie, sa résolution à été menée à l'aide de la méthode des éléments finis 
(MEF) et a permis le calcul des paramètres électriques $[L]$, [C] et $[R]$, à partir de la distribution du potentiel dans le résonateur.

A partir des différentes simulations que nous avons pu mener, nous constatons que l'écart est faible entre nos résultats numériques et ceux trouvés par la méthode BEM. Nous disposons donc d'un outil informatique précieux pour la caractérisation des paramètres primaires du résonateur en cage d'oiseau.

Le modèle numérique étant général. Ici, nous l'avons appliqué au cas du résonateur TEM en cage d'oiseau à 12 éléments pour pouvoir comparer nos résultats obtenus par MEF à ceux obtenus par BEM les seuls à notre disposition. Cet outil numérique peut donc être étendu pour l'analyse de toute forme de structure TEM.

\section{Références}

[1] - F. Taulelle, D. Massiot, J. C. Rifflet, J. P. Coutures, Bulletin magnetic resonance, 11 (1990) 314-316

[2] - A. Leroy-Willing, L. Darasse, J. Taquin, M. Sauzade, Magnetic resonance in medicine, 2 (1985) 20-28

[3] - G. Bogdanov and R. Ludwig, Magnetic Resonance in Medicine, 47, (2002), 579-593

[4] - J. W. Pan, J. T. Vaughan, R. I. Kuzniecky, G. M. Pohost, Magnetic Resonance Imaging, 13 (1995) 915-921

[5] - www . freeFEM . org

[6] - N. Ben Ahmed, M. Feham, M. Kameche, Applied Microwave \& Wireless, 12(10), (2000) 28-38

[7] - N. Ben Ahmed and M. Feham, Microwave Journal, 43 (11) (2000) 106-120

[8] - A. R. Djordjevic, D. Darco, M.C. Goran, T.K. Sarkan, "Artech Housse", (1997). 